\title{
Alternative Treatment for Infectious Arthritis in a Complicated Diabetes Mellitus Patient with Ankle Joint Instability
}

\author{
Yunhae Lee (D), Myungchul Lee (D) \\ Department of Plastic and Reconstructive Surgery, Konkuk University Medical Center, Seoul, Korea
}

\begin{abstract}
Treatment of diabetic foot infections requires attention to both local and systemic issues, as diabetes mellitus is accompanied by a high incidence of complications that delay wound healing and restrict treatment options. We present a patient with diabetes who had infectious arthritis of the ankle joint with a soft tissue defect involving the joint capsule. The comorbidities of the patient limited the options for treatment in reconstruction of the ankle joint defect. Therefore, nonabsorbable sutures bridging the periosteum of the fibula and calcaneus bones were applied to mimic the calcaneofibular ligament and secure the joint capsule. In addition, negative pressure wound therapy (NPWT) was administered to protect the joint space, absorb extra fluid, and promote wound healing. Acellular dermal matrix was used in combination with NPWT to enhance wound healing and tissue regeneration. Although methicillin-resistant Staphylococcus aureus was cultured from the wound several times, there were no signs that this treatment aggravated the infection. After 7 months of inpatient treatment, stable soft tissue coverage and ankle joint stabilization were achieved, allowing fair standing balance, gait and plantar flexion.
\end{abstract}

Keywords: Diabetic foot; Arthritis, infectious; Joint instability

\section{Introduction}

Diabetes mellitus (DM) is a significant clinical condition accompanied by a high incidence of disease-related complications such as neuropathy, nephropathy and cardiovascular diseases [1]. A well-known consequence of DM is insensitivity of the foot that results in unnoticed injuries and delayed initial proper wound care. In addition, comorbidities disturb the normal healing potential of the body and can limit treatment options. Therefore, reconstruction of each wound should be achieved in consideration of both the wound itself and the patient's condition. Here, we report a case involving a DM patient who presented with infectious arthritis of the ankle joint and a soft tissue defect involving the joint capsule. By using nonabsorbable sutures, negative pressure wound therapy (NPWT) and acellular dermal matrix (ADM), joint stability with soft tissue coverage was restored. The study protocol conformed to the ethical guidelines of the Declaration of Helsinki, and a verbal informed consent was obtained from the patient according to the decision of the Konkuk University Medical Center Institutional Review Board (IRB exemption No. KUMC 2019-10-046).

\section{Case}

A 57 year-old male patient with a 20-year history of DM presented with an open wound on the left ankle (lateral malleolar area). He had undergone incision and

\section{Case Report}

Received: October 31, 2019

Revised: November 4, 2019

Accepted: November 4, 2019

\section{Corresponding author:}

Myungchul Lee, M.D.

Department of Plastic and Reconstructive Surgery, Konkuk University Medical Center, 120-1 Neungdong-ro, Gwangjin-gu, Seoul 05030, Korea

Tel: +82-2-2030-8191

Fax: +82-2-2030-5249

E-mail: 20150136@kuh.ac.kr

This is an Open Access article distributed under the terms of the Creative Commons Attribution Non-Commercial License (https://creativecommons.org/licenses/by-nc/4.0/) which permits unrestricted non-commercial use, distribution, and reproduction in any medium, provided the original work is properly cited.

C) 2020 Korean Wound Management Society 
drainage of an abscess formed in the left ankle in another hospital, after which the wound had not healed for 2 months. The wound was $2 \times 6 \mathrm{~cm}$ in size and was marked as Wagner grade 3 . The wound was covered with excessive granulation tissue; however, probing the depth of the wound we encountered a solid surface assumed to be a bony structure. We also noted instability of the ankle joint and discharge was evident with internal rotation (Fig. 1). Infection markers on initial presentation were as follows: white blood cell count, $5.90 \times 10^{3} / \mu \mathrm{L}$; erythrocyte sedimentation rate, $96 \mathrm{~mm} / \mathrm{hr}$; and C-reactive protein, $21.61 \mathrm{mg} / \mathrm{dL}$. The markers of wound healing were as follows: hemoglobin ( $\mathrm{Hb}) \mathrm{A} 1 \mathrm{c}, 5.8 \%$; $\mathrm{Hb}, 8.6 \mathrm{~g} / \mathrm{dL}$; and albu$\min , 3.8 \mathrm{~g} / \mathrm{L}$. Plain radiography showed erosion of the talomalleolar joint (Fig. 2) and 3-phase bone scans could not rule out osteomyelitis. Enhanced foot magnetic resonance imaging showed a skin and soft tissue defect leading into the talocrural joint and an extensive bone marrow signal change and enhancement in the lateral malleolus, distal tibia and talus (Fig. 3). Methicillin-resistant Staphylococcus aureus was identified in the fluids from the affected joint, indicating infectious arthritis.

We applied a removable splint to immobilize the joint and started intravenous antibiotics with $1.5 \mathrm{~g}$ of ampicillin-sulbactam every 12 hours. Once the excessive granulation tissue was removed by surgical debridement, the bony structure of the ankle joint was exposed. We could visualize the damaged synovial capsule and turbid discharge from within the ankle joint when it was internally rotated (Fig. 4). We performed
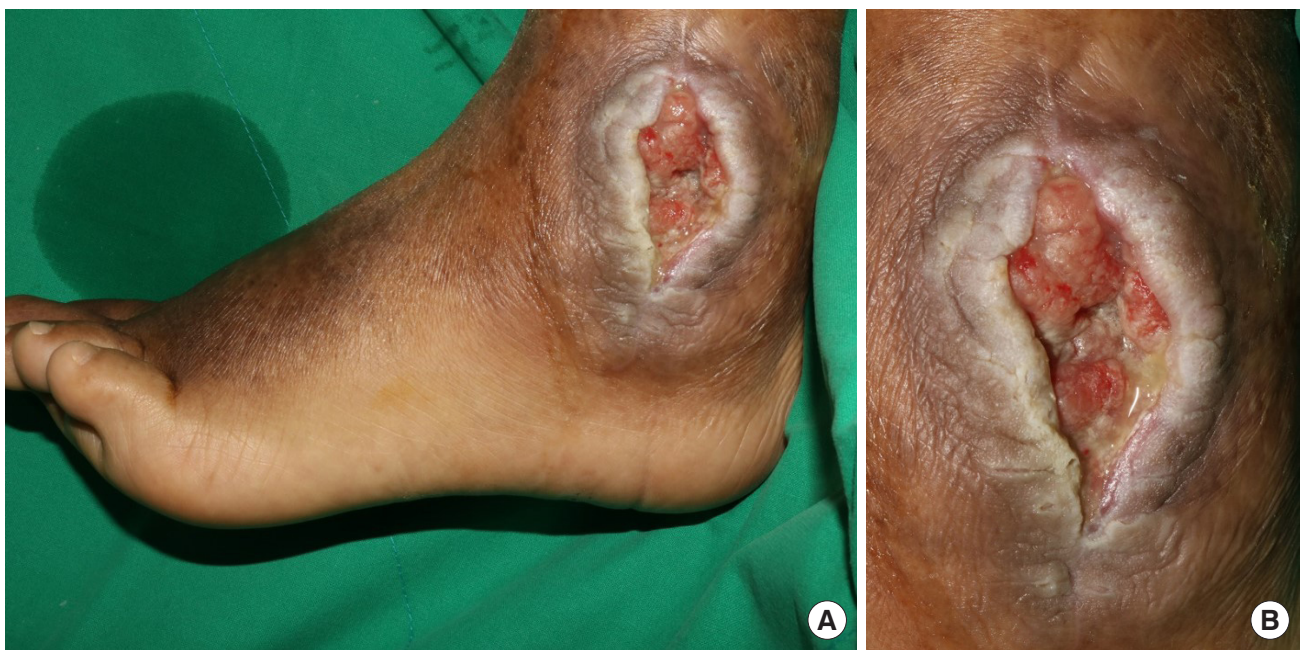

Fig. 1. The initial wound. (A) Note the wound with granulation tissue, marked as Wagner grade 3. (B) Close-up view.
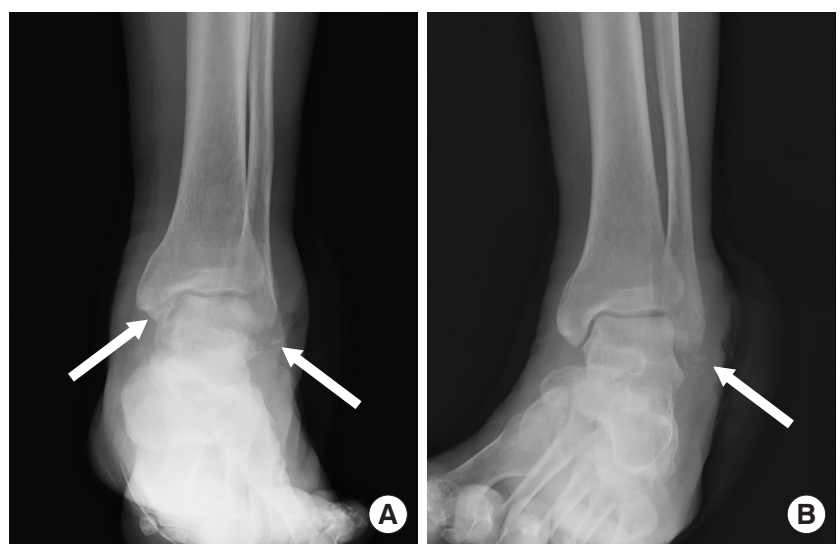

Fig. 2. Anteroposterior and mortise view of ankle radiography. (A, B) Note the bone erosion of the talus and lateral malleolus (white arrows).

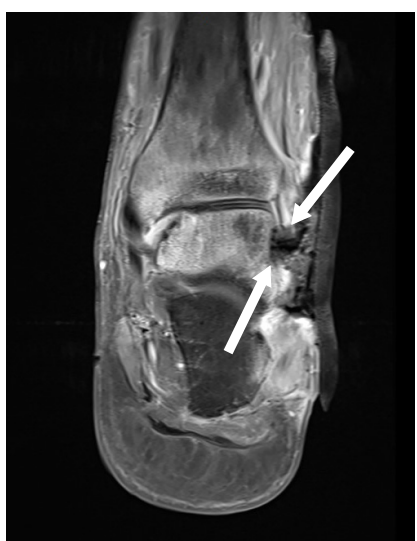

Fig. 3. Coronal view of T2-weighted foot MRI. Note the tissue defect extending into the talocrural joint (white arrows). MRI, magnetic resonance imaging. 

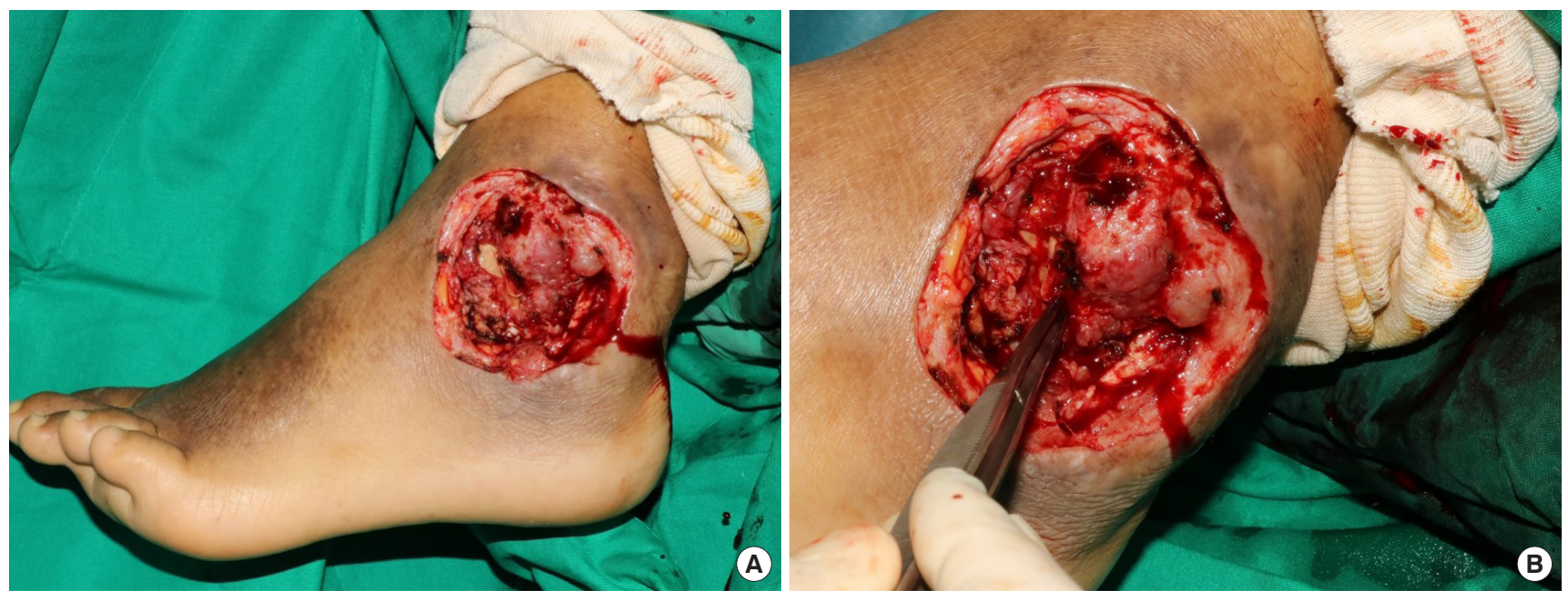

Fig. 4. Postoperative photographs of the wound after debridement. (A, B) Note the open ankle joint indicated with the tip of a forceps.
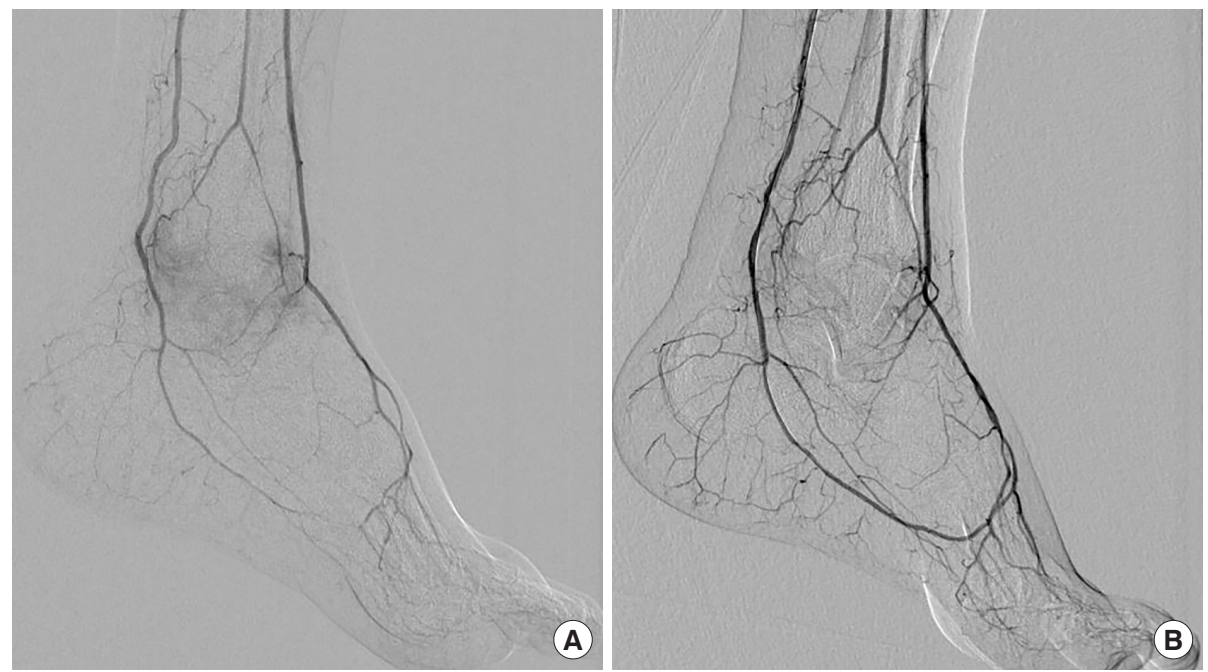

Fig. 5. Angiogram of blood flow below the ankle. (A) Note scanty blood flow before percutaneous transluminal balloon angioplasty. (B) Circulation was improved after the procedure.

surgical debridement two more times. After a week of daily curettage and irrigation in the operation room, no bacterial growth was seen on 4 serial tissue and fluid cultures. We then started applying NPWT to shield the ankle joint and absorb extra fluid. We replaced the device every 3-4 days.

For management of the damaged ankle joint capsule, we consulted with the orthopedic surgery department. As they recommended fusion of the ankle joint, we planned a free tissue transfer for coverage of any foreign material used in the fusion surgery, and reconstruction of the soft tissue of the lateral ankle.

However, the patient's general condition contraindicated this relatively aggressive surgery. The patient had a variety of chronic medical conditions including hypertension, coronary artery disease with a prior silent myocardial ischemic event and $90 \%$ stenosis in the left anterior descending artery, peripheral vascular disease, and chronic renal insufficiency requiring hemodialysis. Further, computed tomography angiography of the legs on initial work-up revealed stenotic lesions requiring percutaneous transluminal balloon angioplasty. After angioplasty, substantial blood flow was observed below the ankle (Fig. 5). These circulatory and renal issues presented an obstacle to free flap surgery as volume overload and inotropics, frequently used for flap survival, could not be administered. In addition to possible flap failure, there was risk of cardiac crisis on the operating table. 

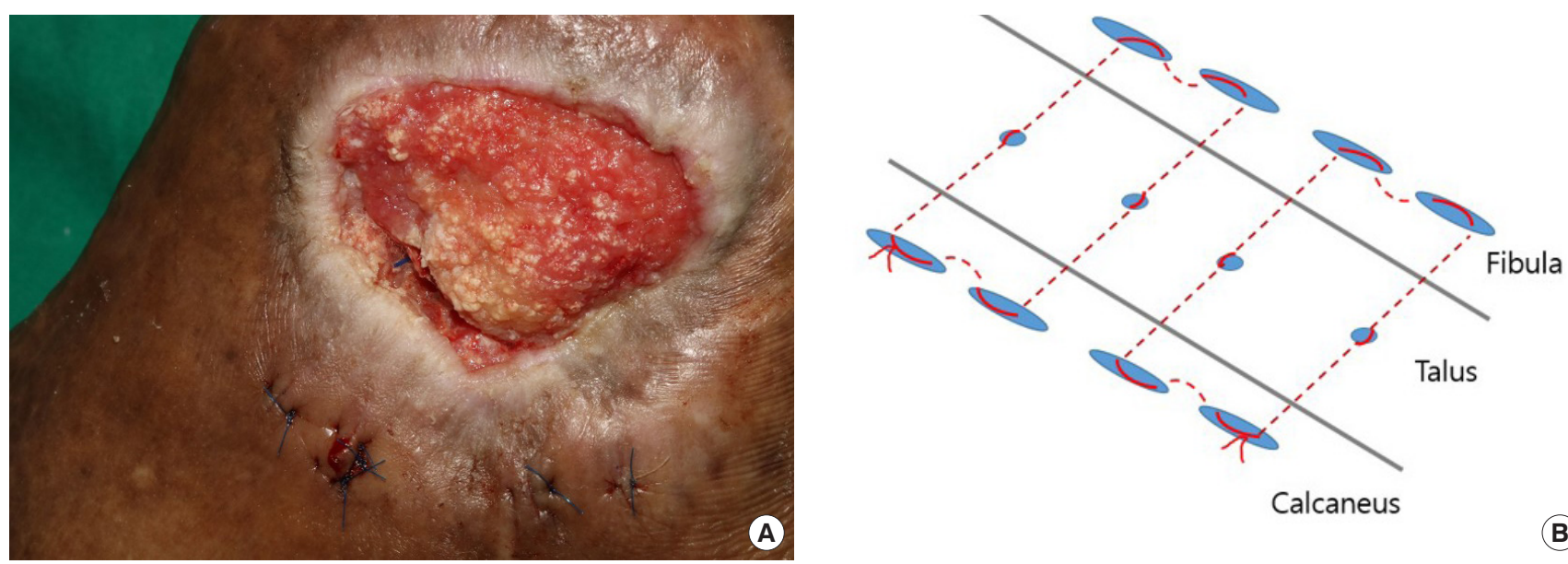

Fig. 6. Surgical technique to stabilize the ankle joint. (A) Postoperative photographs of the wound after applying nonabsorbable sutures. (B) In the illustration, dotted lines indicate the buried portion of the sutures.
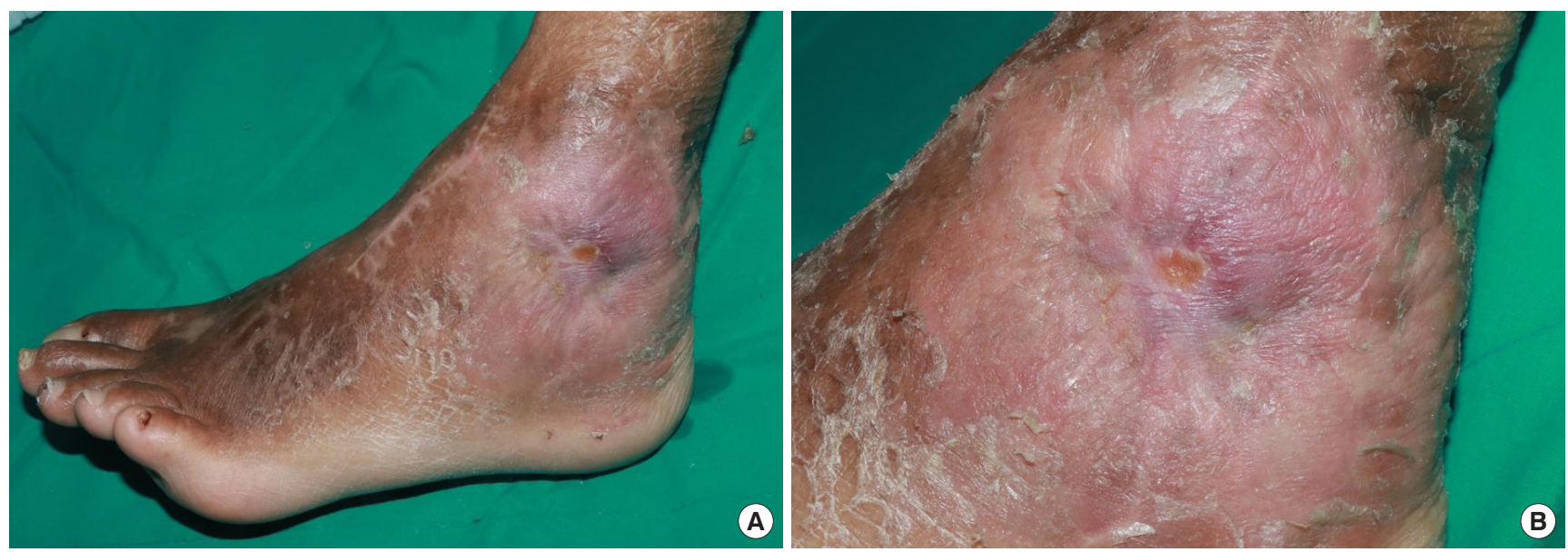

Fig. 7. The healed wound. (A) Complete healing was achieved after 7 months of wound management. (B) Close-up view.

We concluded that without sufficient tissue coverage, foreign materials might become a source of infection or other complications. This called for an alternative method involving minimal use of foreign material that could be performed under local anesthesia.

Nonabsorbable Prolene 1-0 sutures were applied in a buried loop fashion to bridge the fibular and calcaneal bone periosteum so that it would function similar to the calcaneofibular ligament and possibly restore stability to the ankle joint. After slit incisions were made in the periosteum of the fibula, talus, and calcaneus, the buried suture loop was started from the calcaneus towards the fibula and then back to the calcaneus with the needle inserted deep enough to touch the cortical bone (Fig. 6). The knots were buried under the periosteum.

For reconstruction of the soft tissue coverage, we applied a paste-type ADM product (CG paste; CGBio, Seongnam, Ko- rea) with NPWT on the overlying wound of the joint. This treatment was administered every $3-5$ days.

Intravenous antibiotics were administered throughout the entire treatment period. The regimen was later replaced in consultation with the infectious diseases department based on results from the wound cultures carried out between treatment sessions. The initial ampicillin-sulbactam therapy was first changed to $400 \mathrm{mg}$ of teicoplanin every 48 hours, then changed again to $1 \mathrm{~g}$ of vancomycin, once every 3 days. When culture results showed change, we maintained NPWT and $\mathrm{ADM}$ and carried out daily irrigation in the operating room to check for signs of infection, including turbid discharge and fever. Treatment was resumed when there were no obvious clinical signs of infection. We found no meaningful correlation with infection markers and wound culture results.

After 7 months of inpatient treatment, complete healing of 
the wound was observed (Fig. 7), and the patient was able to walk with normal shoes and showed fair standing balance and grade 4 ankle plantar flexion.

\section{Discussion}

The treatment of end-stage arthritis of the ankle and subtalar joint using plates, screws, intramedullary nails, and external fixation is amply described in the literature [2]. Fusion is achieved with good blood supply and appropriate soft tissue conditions. However, in this case, the open wound exposing the ankle joint components would not have provided sufficient soft tissue coverage for the foreign materials used for fusion. Though recruitment of free tissue from proximal parts of the body with relatively large vessels would have been ideal for sufficient coverage of the foreign material and reconstruction of the defect, it was not feasible in this case because of the patient's comorbidities. Although restoration of macrocirculation was observed on post-angioplasty angiograms, macrocirculation does not always lead to microcirculation, which is critical in wound healing. We had doubts as to whether the blood flow was sufficient.

Moreover, there are studies reporting adverse effects of these fusion methods in patients with diabetes, owing to the increased risk of postoperative complications. The nonunion rates (odds ratio, 7-10), infection rates, and incidence of other complications were reported to be higher in patients with diabetes than in patients without diabetes $[3,4]$.

We concluded that an alternative method involving minimal use of foreign material to stabilize the ankle joint capsule was required. To control chronic lateral ankle instability, we could attempt other methods like direct repair or anatomical reconstruction with autografts or allografts [5]. However, degenerative changes to the normal ligamentous structures of the ankle joint were apparent during the delayed wound healing period, making direct repair impossible. Allografts would have added more foreign material, increasing the risk of inflammation. For this case, we borrowed the idea of approximating the periosteum to strengthen the lateral ligamentous structures as described by Ahlgren and Larsson [6]. Their original method approximated periosteum with the attached anterior talofibular and calcaneofibular ligaments and the inferior retinaculum. In our case, to avoid the risk of aggravating ankle instability, we did not perform additional dissection to explore the preservation of these structures. Though we did not confirm the viability of each ligament, we still maintain that restoration of lateral ankle stability was needed because the patient was suffering severe pain and instability in the standing position. To minimize the burden of foreign material, we used a buried loop technique and hid the knots under the periosteum.

We applied NPWT from the early stages of treatment for several reasons. First, we wanted to seal and provide a moist environment to the open ankle joint and also needed a means to absorb the extra synovial fluid to prevent fluid collection and suppress bacterial colonization. NPWT also had the additional benefit of accelerating wound healing and closure. Evidence supporting the use of NPWT in the treatment of diabetic foot wounds includes numerous prospective and multi-centered randomized controlled trials $[7,8]$. NPWT is thought to improve dermal blood flow through vasomotor mediators, accelerate wound contraction and size reduction by direct macrodeformation, and induce granulation and angiogenesis and reduce edema, all of which were relevant to our case [9].

After the use of NPWT and proper management of necrotic tissues between each session, we observed substantial capillary bleeding from the wound bed. We therefore presumed that microcirculation was adequate for continued treatment. We believe that observation of capillary bleeding, however subjective, is one of the easiest ways to evaluate microcirculation and we encourage surgeons to check for capillary bleeding prior to using any methods described in this paper.

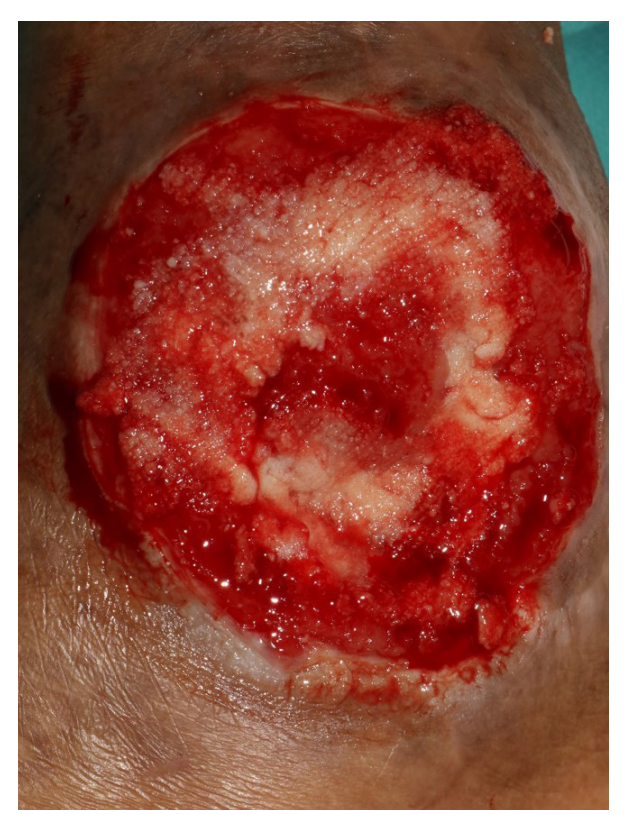

Fig. 8. Paste-type ADM applied on the wound. Note the joint space fully filled with ADM. ADM, acellular dermal matrix. 
To further accelerate wound healing, we used a combination of ADM and NPWT. The paste-type ADM used in our case is a mixture of micronized ADM and gelatin. The paste form of the mixture allows it to conform to the shape of the containing environment, thus enabling full contact, even for narrow spaces (Fig. 8). This creates maximum surface area for the ADM to accelerate wound healing as an extracellular matrix for tissue regeneration.

Despite the healing potential of ADM, we must be aware of the possible adverse events. In our previous experiences with this specific ADM, we have observed minor fluid collection. We are not sure whether this was liquefaction of the ADM material or fluid inflow from the surrounding tissues. For the case at hand, we watched for fluid accumulation as a possible source of bacterial colonization or inflammation. A metaanalysis of randomized controlled trials by Guo et al. [10] compared the efficacy and safety of ADM to standard of care in diabetic foot ulcer patients. They reported no significant differences in the adverse events of infection, amputation, seroma, and immune response between the ADM and standard care group during the follow-up (relative risk, 0.98). In light of this meta-analysis and our experience, we recommend using $\mathrm{ADM}$ with caution, only after infection control is evident and with ongoing cultures to identify signs of infection and inflammation. In addition, clinicians should consider concurrent use of NPWT to absorb fluid accumulation.

In conclusion, our experience suggests that $\mathrm{ADM}$ and NPWT combined with autologous repair of the joint can provide a basis for stable soft tissue coverage and joint stability, achieving limb salvage in complex ankle joint wounds in medically compromised patients.

\section{Conflict of interest}

No potential conflicts of interest relevant to this article are reported.

\section{ORCID iDs}

$\begin{array}{ll}\text { Yunhae Lee } & \text { https://orcid.org/0000-0002-7801-8898 } \\ \text { Myungchul Lee } & \text { https://orcid.org/0000-0002-9721-0092 }\end{array}$

\section{References}

1. Tijdens-Creusen EJA, Jutte PC, Schoumakers WMHH, et al. Orthopedic aspects of the diabetic foot. Curr Pharm Des 2018;24:1255-69.

2. Carpenter B, Thomas J, Brigido SA, et al. Tibio-talar-calcaneal fusion in the diabetic and nondiabetic patient: an update on surgical techniques. Foot Ankle Spec 2019;12:1724.

3. Lee M, Choi WJ, Han SH, et al. Uncontrolled diabetes as a potential risk factor in tibiotalocalcaneal fusion using a retrograde intramedullary nail. Foot Ankle Surg 2018;24:5428.

4. Gross JB, Belleville R, Nespola A, et al. Influencing factors of functional result and bone union in tibiotalocalcaneal arthrodesis with intramedullary locking nail: a retrospective series of 30 cases. Eur J Orthop Surg Traumatol 2014; 24:627-33.

5. Yasui Y, Murawski CD, Wollstein A, et al. Operative treatment of lateral ankle instability. JBJS Rev 2016;4:01874474201605000-00006.

6. Ahlgren $\mathrm{O}$, Larsson S. Reconstruction for lateral ligament injuries of the ankle. J Bone Joint Surg Br 1989;71:300-3.

7. Vig S, Dowsett C, Berg L, et al. Evidence-based recommendations for the use of negative pressure wound therapy in chronic wounds: steps towards an international consensus. J Tissue Viability 2011;20 Suppl 1:S1-18.

8. Eginton MT, Brown KR, Seabrook GR, et al. A prospective randomized evaluation of negative-pressure wound dressings for diabetic foot wounds. Ann Vasc Surg 2003;17:6459.

9. Hasan MY, Teo R, Nather A. Negative-pressure wound therapy for management of diabetic foot wounds: a review of the mechanism of action, clinical applications, and recent developments. Diabet Foot Ankle 2015;6:27618.

10. Guo X, Mu D, Gao F. Efficacy and safety of acellular dermal matrix in diabetic foot ulcer treatment: a systematic review and meta-analysis. Int J Surg 2017;40:1-7. 